\title{
Desenvolvimento urbano sustentável e qualidade de vida: uma pesquisa bibliométrica das publicações na base Web of Science
}

\section{Pabla Pereira da Silva, Roberto Schoproni Bichueti, Carlos Rafael Röhrig da Costa, Gabriela Dubou, Estela dos Anjos Pires*}

Universidade Federal de Santa Maria. Av. Roraima, 1000 - 7. Camobi. Santa Maria-RS, Brasil (CEP 97105-900).*E-mail: estelaanjpires@gmail.com.

Resumo. Este estudo tem como objetivo analisar as características da produção científica relacionadas às temáticas desenvolvimento urbano sustentável (sustainable urban development) e qualidade de vida (quality of life), por meio da base de dados Web of Science (WoS), no período de 2008 a 2017. Para isso, o estudo possui uma abordagem quantitativa e qualitativa, onde a etapa quantitativa buscou identificar algumas variáveis acerca da produção científica sobre Desenvolvimento Urbano Sustentável e Qualidade de Vida e a etapa qualitativa buscou analisar os conteúdos presentes nos dez artigos mais citados, de acordo com o relatório de citações da Web of Science. A partir da pesquisa realizada, pode-se constatar que os estudos sobre desenvolvimento urbano sustentável e qualidade de vida são emergentes e estão em constante evolução. Além disso, foi possível verificar que as temáticas pesquisadas consistem em assuntos multidisciplinares. Por fim, ao analisar os dez artigos mais citados, concluiu-se que as suas contribuições são de grande relevância para os estudos futuros em relação ao desenvolvimento sustentável das cidades, bem como a qualidade de vida dos cidadãos.

Palavras-chave: Desenvolvimento urbano sustentável; Qualidade de vida; Pesquisa bibliométrica.

Abstract. Sustainable urban development and quality of life: $A$ bibliometric research of publications on the web of science. This study aims to analyze the characteristics of scientific production related to sustainable urban development and quality of life, through the Web of Science (WoS) database, from 2008 to 2017. For this, the study has a quantitative and qualitative approach. The quantitative step, sought to identify some variables about scientific production on Sustainable Urban Development and Quality of Life, and the qualitative step sought to analyze the content present in the ten most cited articles, according to the Web of Science citation report. From the research carried out, it can be seen that the studies on sustainable urban development and quality of life are emerging and are constantly evolving. In addition, it was possible to verify that the subjects researched
Recebido:

$25 / 07 / 2018$

Aceito:

$23 / 08 / 2018$

Publicado:

$31 / 08 / 2018$

Acesso aberto

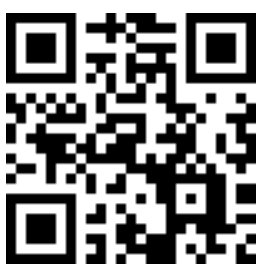

ORCID

(ㄷ) 0000-0002-0364-4034 Pabla Pereira da Silva

(1) 0000-0002-7548-720X Roberto Schoproni Bichueti

(1) 0000-0002-0721-1615 Carlos Rafael Röhrig da Costa

(1) 0000-0003-0813-521X Gabriela Dubou

D) 0000-0002-8720-9354 Estela dos Anjos Pires 
consist of multidisciplinary subjects. Finally, in analyzing the ten most cited articles, it was concluded that their contributions are of great relevance to future studies on the sustainable development of cities, as well as the quality of life of citizens.

Keywords: Sustainable Urban Development; Quality of life; Bibliometric Research.

\section{Introdução}

O aumento da migração para as cidades, o envelhecimento da população e os impactos nas mudanças climáticas são alguns dos desafios que $o$ desenvolvimento urbano sustentável procura resolver em todo o mundo. Em 2009, havia 21 megacidades no mundo, cada uma com pelo menos 10 milhões de habitantes, representando $9,4 \%$ da população urbana mundial. Prevê-se que o número de megacidades aumentará para 29 em 2025, e serão habitadas por $10,3 \%$ da população urbana mundial (Han et al. 2012).

Além disso, os impactos das mudanças climáticas também estão sendo sentidos nas cidades. Algumas cidades estão localizadas em áreas baixas, perto de litorais e rios, totalmente vulneráveis a riscos potenciais devido às alterações climáticas, tais como tufões e ciclones intensos, aumento do nível do mar, ondas extremas e surtos de tempestades (IPCC, 2007).

Quanto ao envelhecimento, Han et al. (2012) explica que vidas mais longas também podem resultar em aumento dos custos médicos e mais demandas por serviços e instalações de saúde, já que as pessoas mais velhas são mais suscetíveis a doenças. E mais, os esquemas de transporte e habitação devem ser modificados para atender a um número crescente de pessoas mais velhas (Bartley e O'Neill, 2010).

Diante desses problemas, tornase vital pensar em soluções sustentáveis para o futuro das cidades. Além do mais, problemas típicos da grande cidade, como a segregação, a degradação da vizinhança, o aumento do tráfego rodoviário, a privação socioeconômica e as desigualdades na saúde e no bemestar tornaram-se questões políticas centrais na maioria dos países (Kamp et al., 2003).

De acordo com o Programa Cidades Sustentáveis (2018), a gestão urbana deve atuar no sentido de assumir plenamente as responsabilidades para proteger, preservar e assegurar o acesso equilibrado aos bens naturais comuns. Segundo Giddings et al. (2005), as cidades podem proporcionar uma rica e variada qualidade de vida a todos os seus habitantes, mas isso depende de uma mudança na forma como são financiadas e governadas.

Assim sendo, o desenvolvimento sustentável das cidades busca, entre outros objetivos, desenvolver soluções para elevar a qualidade de vida dos habitantes. Entre as condições que moldam a qualidade de vida, de acordo com Wahl e Weisman (2003), o meio ambiente desempenha um papel significativo na criação de uma boa vida. 0 que aumenta o bem-estar das pessoas é a harmonia entre preferências pessoais e características ambientais (Kilinc, 2006).

Neste contexto, tendo em vista evidências de relação entre a qualidade de vida e o desenvolvimento urbano sustentável, o presente trabalho busca analisar as características da produção científica relacionadas às temáticas. Para tanto, desenvolveu-se um estudo bibliométrico, por meio da base de dados Web of Science, no período de 2008 a 2017, dos tópicos "desenvolvimento urbano sustentável" (sustainable urban development) e "qualidade de vida" (quality of life). Também procurou-se identificar e analisar as publicações mais 
relevantes dos temas, a partir do levantamento dos dez estudos mais citados ao longo deste período. Por meio dessa análise, pretende-se ampliar 0 conhecimento acerca das temáticas, bem como, compreender o estado-da-arte desse campo de estudo, tendo em vista a ampla gama de possibilidades de assuntos que os temas envolvem.

Para alcançar o objetivo proposto, o estudo se inicia com o aporte teórico referente ao desenvolvimento urbano sustentável e a qualidade de vida. Em seguida, apresenta-se o método do estudo. Posteriormente, são descritos os resultados obtidos na análise da produção científica internacional envolvendo as temáticas do trabalho.

\section{Referencial teórico}

\section{Desenvolvimento urbano sustentável}

A conquista de cidades sustentáveis requer um conjunto de ações que procuram atender as mais diversas áreas e necessidades que um espaço urbano exige. De acordo com Oliveira e Souza-Lima (2006), pensar em desenvolvimento é, antes de qualquer coisa, pensar em distribuição de renda, saúde, educação, ambiente, liberdade, lazer, entre outras variáveis que possam afetar a qualidade de vida da sociedade.

Segundo Williams (2010), os dois principais desafios para o desenvolvimento urbano sustentável são: (1) compreender a 'visão', ou seja, saber o que realmente se entende por cidade sustentável, e (2) desenvolver uma compreensão mais profunda dos processos multifacetados de mudança necessários para alcançar cidades mais sustentáveis.

Neste sentido, dado os desafios para amenizar os efeitos negativos do crescimento urbano e direcionar as cidades ao desenvolvimento sustentável, entende-se, conforme Kobayashi et. Al (2006), uma cidade sustentável como um lugar que atende aos objetivos sociais, ambientais, políticos e culturais, bem como aos objetivos econômicos e físicos de seus cidadãos. E ainda, seus recursos devem ser utilizados da forma mais eficiente possível para atender tais objetivos.

Além disto, Rogers (2013) coloca
que o conceito de cidade
autossustentável reinventa e revisita o modelo de cidade densa. Ele explica que o modelo compacto foi rejeitado no Século XX, em função de problemas como a superpopulação, pobreza, esgoto a céu aberto, entre outros. Entretanto, com sistemas mais avançados de tratamento de esgoto e do lixo, a disponibilidade de produtos ecologicamente corretos e sistemas mais avançados de energia e transporte públicos, o modelo de cidade densa pode ser reconsiderado, em especial em função das vantagens sociais da proximidade (Rogers, 2013).

Leite e Awad (2012) também defendem que as cidades sustentáveis devem ser densas e compactas. Os autores acreditam que para conquistar metrópoles compactas, densas e vivas é preciso focar na regeneração urbana e na reestruturação produtiva de áreas metropolitanas.

Caminhando com esse conceito de cidades densas e compactas está o conceito de cidade inteligente. De acordo com Duran e Perez (2015), em todo o mundo, está crescendo a implementação do modelo de cidades inteligentes com desenvolvimento sustentável, conceito orientado a aperfeiçoar o investimento social, o capital humano, comunicações, infraestrutura, assegurando a sinergia entre cada um dos elementos que o compõem.

Conforme Cortese et al. (2017), uma cidade inteligente é aquela capaz de criar estruturas de gestão qualificadas para atender demandas próprias do caráter problemático que o espaço urbano, enquanto sistema complexo, produz continuamente. Portanto, uma cidade inteligente faz uso da tecnologia de informação para otimizar tarefas e tornar mais eficiente os serviços públicos. 
Em meio às soluções pensadas e apresentadas para a conquista de uma cidade sustentável, percebe-se a preocupação em elevar a qualidade de vida da comunidade. O relatório das Nações Unidas (2017) afirma que políticas integradas para melhorar a vida dos moradores urbanos e rurais são extremamente necessárias. Segundo Giddings et al. (2005), as cidades podem proporcionar elevada qualidade de vida aos seus habitantes, operando em uma escala humana, nas quais os espaços e lugares sejam dedicados a interação social e aos negócios diários da vida.

Rogers (2013) destaca que a qualidade de vida da população é um dos alicerces de uma cidade sustentável. Corroborando, Gehl (2013) informa que a preocupação com a dimensão humana no planejamento urbano é crescente e reflete uma exigência distinta e forte por melhor qualidade de vida urbana.

Diante do contexto apresentado, a próxima seção segue com reflexões sobre o conceito de qualidade de vida e a sua relação com o desenvolvimento urbano sustentável.

\section{Qualidade de vida}

A relação entre meio ambiente urbano e qualidade de vida é pensada levando-se em conta aspectos estreitamente ligados a uma abordagem intersetorial da questão (Jacobi, 2000). Assim, como o desenvolvimento urbano sustentável, a qualidade de vida também abrange vários setores.

No campo conceitual, a mescla entre os dois conceitos (qualidade de vida e qualidade ambiental) é de tal ordem, que muitas vezes se torna difícil estabelecer se a qualidade de vida é um dos aspectos da qualidade ambiental, ou se a qualidade ambiental é um componente do conceito de qualidade de vida (Kran e Ferreira, 2006). Na prática, os autores explicam que a abrangência de cada um dos conceitos se explicita, ao serem estabelecidos e aplicados os métodos para a sua mensuração, que acabam, assim, contribuindo para a formação do próprio conceito.

Como ressalta Ülengin et al. (2001), diferentes resultados são obtidos nos estudos de qualidade de vida, devido as diferenças na escolha dos conjuntos de variáveis, na atribuição de pesos às variáveis, da abordagem adotada, das metodologias usadas, e a homogeneidade das unidades geográficas de análise em que as pesquisas são baseadas.

No que se refere ao desenvolvimento urbano sustentável, a qualidade de vida abrange dimensões econômicas, social e ambiental. As cidades podem ser consideradas como pacotes de serviços prestados aos cidadãos, onde suas necessidades e desejos correspondem a aspectos sociais e econômicos da cidade. Essas necessidades devem ser observadas no planejamento urbano, projetado para melhorar a qualidade de vida das pessoas (Węziak-Białowolska, 2016).

Desse modo, Zapata e Romero (2010) salientam: embora a qualidade de vida possa ser determinada por fatores objetivos, sua manifestação essencial é apreciada na ordem subjetiva, resposta de uma relação dialética entre o objetivo e o subjetivo. Sendo assim, apesar da complexidade do tema, Vizcaíno e Fernández (2009) advertem que há três premissas a serem consideradas para avaliação: a primeira diz respeito à satisfação reconhecida pelo indivíduo, o segundo às condições de vida em que a pessoa subsiste e a terceira a uma experiência percebida dos dois anteriores.

Além disso, a qualidade de vida é construída social e coletivamente e os próprios indivíduos interferem na sua construção considerando os variados cenários pessoais, familiares, institucionais e comunitários. As pessoas, especificamente os residentes, percebem as características ambientais de acordo com a relevância de suas necessidades (Kahana et al., 2003).

A qualidade de vida, de acordo com Tovar (2010), é plural e não 
singular, no sentido de que a qualificação da sensação de existência é criada pelo indivíduo com suas múltiplas determinações geradas pela sua história ontogenia. E mais, conforme Turkoglu (2015), aqueles interessados nos estudos sobre cidades e meio ambiente em geral devem estar conscientes de que vivemos em diferentes lugares, cada um dos quais tem numerosos atributos ambientais e esses lugares também são importantes para a nossa qualidade de vida.

A vista do que foi apresentado, pode-se perceber que os estudos sobre qualidade de vida voltada para o desenvolvimento urbano sustentável podem ser encontrados em diferentes áreas de pesquisa, muitas vezes, levando em conta aspectos objetivos e subjetivos para alcançar melhor compreensão das necessidades humanas dentro de uma cidade.

Portanto, torna-se importante analisar as publicações relacionadas às temáticas de desenvolvimento urbano sustentável e qualidade de vida, a fim de ampliar o conhecimento acerca desses temas, bem como, compreender 0 estado-da-arte desse campo de estudo. 0 próximo capítulo apresenta a metodologia usada no presente artigo.

\section{Método de pesquisa}

Inicialmente, destaca-se que este estudo consiste de uma pesquisa bibliométrica, quantitativa, que tem por objetivo identificar as principais características da produção científica internacional relacionada aos temas "qualidade de vida" e "desenvolvimento urbano sustentável", nos últimos dez anos. Neste sentido, desenvolveu-se uma busca na base de dados Web of Science, compreendendo o período de publicações entre 2008 a 2017.

De acordo com Silva (2004), a bibliometria tem o objetivo de analisar a atividade científica ou técnica por meio do estudo quantitativo das publicações. Nesse sentido, um estudo bibliométrico consiste na aplicação dos métodos estatísticos sobre o conjunto de referências bibliográficas e ajuda a conhecer o estágio de evolução em que a pesquisa em determinada área se encontra (Rostaing, 1997; Macedo et al., 2009).

0 estudo também possui uma abordagem qualitativa, tendo em vista que analisa os conteúdos presentes nos dez artigos mais citados, durante o período de 2008-2017. Os dados para a realização desta pesquisa foram coletados através da base Web of Science do Institute for Scientific Information (ISI). A partir do mecanismo de busca disponível na Web of Science, realizou-se a pesquisa com as palavras-chave "desenvolvimento urbano sustentável" (sustainable urban development) $\mathrm{e}$ "qualidade de vida" (quality of life). 0 período de consulta foi restrito aos artigos publicados entre 2008 e 2017, totalizando 10 anos. Os resultados foram analisados em torno de suas principais características, tais como os tipos da produção, os principais autores, instituições, países e agências financiadoras. Analisou-se, ainda, o ano, as fontes dos dados, as áreas temáticas e o idioma das publicações.

A partir da caracterização do estudo, pode-se avançar em direção aos resultados da pesquisa.

\section{Análise dos resultados}

Conforme proposto no objetivo deste estudo, esta seção apresenta as principais características da produção científica internacional relacionada aos tópicos "desenvolvimento urbano sustentável" (sustainable urban development) e "qualidade de vida" (quality of life). Os resultados apresentados referem-se, inicialmente, ao levantamento das principais características da produção científica no período compreendido entre os anos de 2008 e 2017, por meio da pesquisa realizada, em 24 de maio de 2018, na base de dados Web of Science. Em seguida, é apresentada a análise das 
citações dos artigos compreendidos nesta pesquisa.

\section{Características das publicações}

A consulta realizada na base $W e b$ of Science resultou em um levantamento de 528 publicações que relacionam os tópicos 'qualidade de vida' e 'desenvolvimento urbano sustentável'. A partir desse levantamento, serão analisadas as características gerias das publicações de acordo com as seguintes categorias: áreas temáticas das publicações, tipos de documentos, ano das publicações, principais autores, títulos das fontes, principais instituições, idiomas e países das publicações.

Deste modo, pode-se observar que ao longo do período de dez anos (que resultou o número 528 publicações relacionadas aos tópicos citados), a produção científica associada a estas temáticas apresentou um crescimento significativo, passando de 27 publicações no ano de 2008, para 115 estudos em 2017, conforme apresentado na Figura 1, a seguir. Neste período, destaca-se a produção científica do ano de 2015, que apresentou um crescimento significativo, comparado ao ano anterior.

Figura 1. Evolução da produção científica ao longo dos anos.

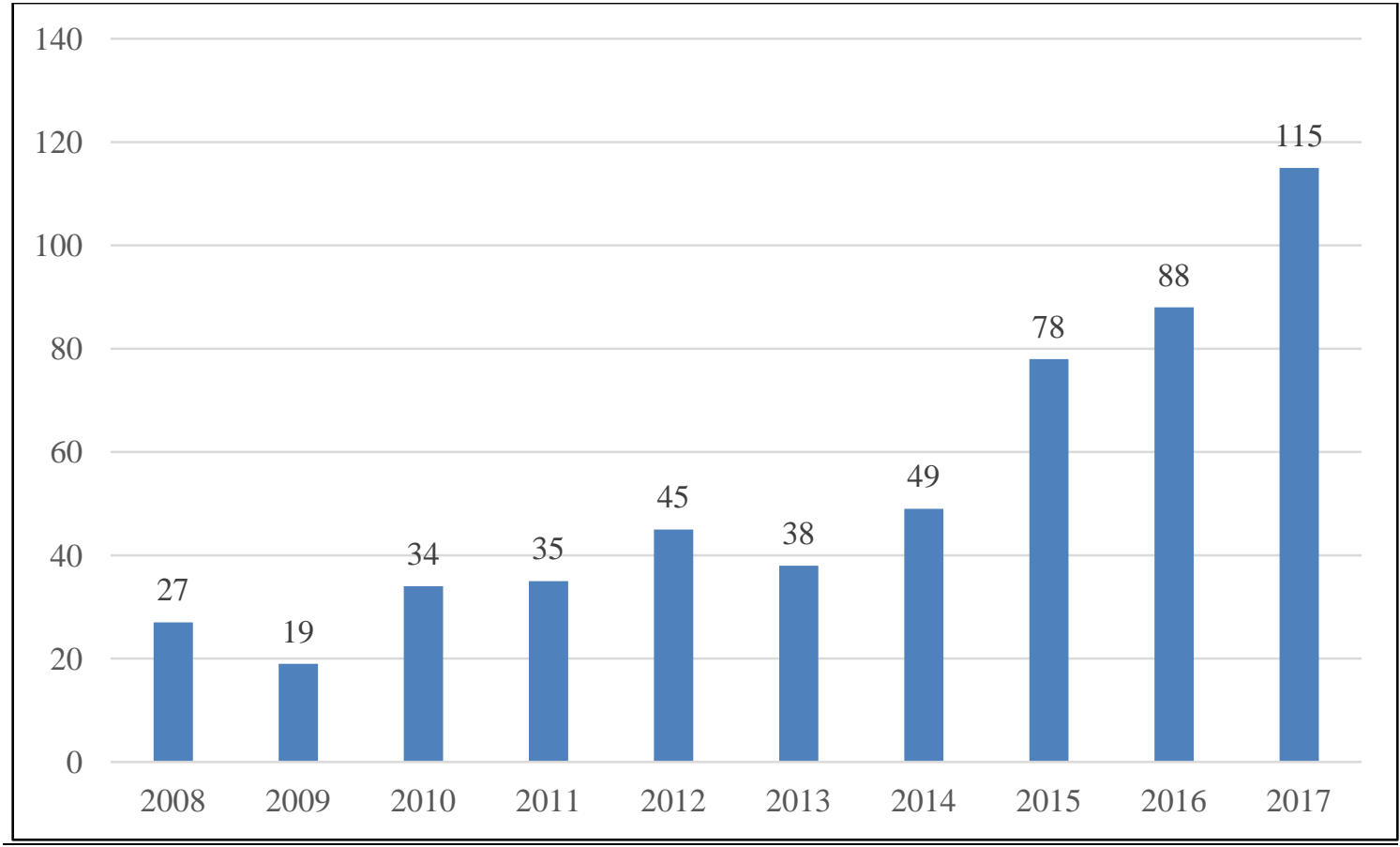

Fonte: Dados da pesquisa - Web of Science

Ressalta-se que grande parte dos estudos são artigos, representando aproximadamente $51 \%$ destes, conforme evidenciado na Tabela 1. Destacam-se, ainda, os papers publicados em anais de congressos, com $46 \%$ dos documentos. Neste sentido, pode-se afirmar que a maior parte destas publicações é considerada produção científica. Tendo em vista que os artigos e os papers em anais são predominantes entre as publicações, torna-se importante ressaltar os títulos das principais fontes de estudos que abordam as temáticas 'qualidade de vida' e 'desenvolvimento urbano sustentável'. Desse modo, a 
Tabela 2 apresenta os nomes dos periódicos e anais de congressos com os maiores números de publicações nesta temática.

Tabela 1. Classificação da produção quanto ao tipo de estudo

\begin{tabular}{llcc}
\hline & Tipo de produção & Publicações $^{\mathbf{1}}$ & \% de 2109 $^{\text {\% }}$ \\
\hline $1^{\text {o }}$ & Artigos & 273 & $51,70 \%$ \\
$2^{\text {o }}$ & Proceedings paper & 245 & $46,40 \%$ \\
$3^{\text {o }}$ & Revisões (review) & 17 & $3,22 \%$ \\
$4^{\text {o }}$ & Materiais editoriais & 1 & $0,19 \%$ \\
\hline
\end{tabular}

${ }^{1}$ Os estudos podem ser classificados em mais de uma categoria.

Fonte: Dados da pesquisa - Web of Science.

Tabela 2. Principais fontes de estudos

\begin{tabular}{|c|c|c|}
\hline & Títulos Da Fonte & Publicações \\
\hline $1^{0}$ & Procedia Engineering & 16 \\
\hline $2^{\circ}$ & Procedia Social and Behavioral Sciences & 14 \\
\hline $3^{\circ}$ & Sustainability & 13 \\
\hline $4 \underline{0}$ & $\begin{array}{l}\text { International Multidisciplinary Scientific Conferences on Social Sciences } \\
\text { and Arts }\end{array}$ & 9 \\
\hline $5^{0}$ & Procedia Environmental Sciences & 7 \\
\hline $6^{0}$ & Habitat International & 6 \\
\hline $7 \underline{0}$ & International Multidisciplinary Scientific Geoconference Sgem & 6 \\
\hline $8^{0}$ & $\begin{array}{l}2011 \text { International Conference on Green Buildings and Sustainable } \\
\text { Cities }\end{array}$ & 5 \\
\hline 9o & Ecological Indicators & 5 \\
\hline $10^{\circ}$ & Fabbrica Della Conoscenza & 5 \\
\hline $11^{\mathrm{o}}$ & Iop Conference Series Earth and Environmental Science & 5 \\
\hline $12^{\mathrm{o}}$ & Journal of Cleaner Production & 5 \\
\hline $13^{0}$ & Social Indicators Research & 5 \\
\hline $14^{\circ} \mathrm{O}$ & Urban Transitions Conference & 5 \\
\hline $15^{0}$ & Wit Transactions on Ecology and the Environment & 5 \\
\hline $16^{0}$ & $\begin{array}{l}\text { 2nd International Symposium New Metropolitan Perspectives Strategic } \\
\text { Planning Spatial Planning Economic Programs and Decision Support } \\
\text { Tools Through the Implementation Of Horizon Europe2020 Isth2020 }\end{array}$ & 4 \\
\hline $17^{0}$ & Applied Mechanics and Materials & 4 \\
\hline $18^{0}$ & Energy Procedia & 4 \\
\hline 190 & European Planning Studies & 4 \\
\hline $20^{\circ}$ & Inted Proceedings & 4 \\
\hline $21^{\mathrm{o}}$ & Journal of Environmental Protection and Ecology & 4 \\
\hline $22^{\circ}$ & Land Use Policy & 4 \\
\hline $23^{0}$ & Sustainable Cities and Society & 4 \\
\hline 240 & Sustainable Development & 4 \\
\hline $25^{0}$ & Transportation Research Procedia & 4 \\
\hline
\end{tabular}

Fonte: Dados da pesquisa - Web of Science. 
Conforme a Tabela 2, encontramse entre as principais fontes os seguintes Journals: Procedia Engineering, Procedia Social and Behavioral Sciences e Sustainability. Ressalta-se que, apesar de cada journal abranger campos de conhecimento diferentes, todos buscam por questões relacionadas ao desenvolvimento urbano sustentável, evidenciando a importância da sustentabilidade e a urbanização para um desenvolvimento favorável em todas as áreas de pesquisa.
Foram analisados, ainda, os principais autores dos estudos nestas temáticas e a quantidade de publicações a eles associados. Conforme descrito na Tabela 3, a seguir, pode-se ressaltar uma pequena concentração da produção científica, na medida em que diversos autores figuram com quantidade relativamente equivalente de publicações.

Tabela 3. Principais autores das publicações.

\begin{tabular}{|c|c|c|c|c|c|}
\hline & Autor & Publicações & & Autor & Publicações \\
\hline $1^{o}$ & Jim CY & 4 & $14^{\circ} \mathrm{O}$ & Fu YJ & 2 \\
\hline $2^{\circ}$ & Yigitcanlar T & 4 & $15^{\circ}$ & Gambardella C & 2 \\
\hline $3^{\circ}$ & Maliene V & 3 & $16^{\circ}$ & Giffinger R & 2 \\
\hline $4 \underline{0}$ & Winston N & 3 & $17^{\circ}$ & Glotz-Richter M & 2 \\
\hline $5^{\circ}$ & Akylas E & 2 & $18^{\circ}$ & Gret-Regamey A & 2 \\
\hline $6^{0}$ & Anopchenko T & 2 & $19^{\circ}$ & Grigonis V & 2 \\
\hline $7^{\circ} \stackrel{0}{2}+2$ & Arquilla V & 2 & $20^{\circ}$ & Griskeviciene D & 2 \\
\hline $8^{\circ}$ & Banzhaf E & 2 & $21^{\circ}$ & Gunter A & 2 \\
\hline 9o & Burinskiene M & 2 & $22^{\circ}$ & Hayek UW & 2 \\
\hline $10^{\circ}$ & Chen WY & 2 & $23^{\circ}$ & Huang XX & 2 \\
\hline $11^{\circ}$ & Crittenden J & 2 & $24^{\circ}$ & Italos C & 2 \\
\hline $12^{\mathrm{o}}$ & Duan L & 2 & $25^{\circ}$ & Jamaludin $\mathrm{N}$ & 2 \\
\hline $13^{\circ}$ & El Menshawy A & 2 & & & \\
\hline
\end{tabular}

Fonte: Dados da pesquisa - Web of Science.

Na Tabela 4, pode-se identificar os países de origem da produção científica envolvendo as temáticas 'qualidade de vida' e 'desenvolvimento urbano sustentável'. Observa-se a predominância dos estudos oriundos da China, Itália e Estados Unidos, e ainda, a presença do Brasil neste ranking, ocupando a $10^{\underline{a}}$ posição entre os países com maior número de publicações nesta área.

Destaca-se que, apesar da
multiplicidade de países e da
superioridade em número de publicações
da China, existe predominância do
idioma inglês nas publicações
internacionais. Conforme apresentado na
Tabela 5, aproximadamente $92 \%$ dos
estudos listados na pesquisa são
disponibilizados neste idioma.

Destaca-se que, apesar da multiplicidade de países e da superioridade em número de publicações da China, existe predominância do idioma inglês nas publicações nternacionais. Conforme apresentado na Tabela 5, aproximadamente $92 \%$ dos disponibilizados neste idioma. 
Tabela 4. Principais países de origem das publicações.

\begin{tabular}{|c|c|c|c|c|c|}
\hline & País & Publicações & & País & Publicações \\
\hline 10 & China & 67 & $14^{\circ}$ & França & 14 \\
\hline $2^{o}$ & Itália & 47 & $15^{0}$ & Canadá & 13 \\
\hline $3^{\circ}$ & Estados Unidos & 47 & $16^{\circ}$ & Egito & 12 \\
\hline $4 \underline{0}$ & Inglaterra & 30 & $17^{\circ}$ & Alemanha & 12 \\
\hline $5^{\circ}$ & Romênia & 27 & $18^{\circ}$ & Grécia & 12 \\
\hline $6^{0}$ & Espanha & 25 & 19 o & Portugal & 12 \\
\hline $7 \underline{0}$ & Polônia & 23 & $20^{\circ}$ & Turquia & 12 \\
\hline $8^{\circ}$ & Índia & 21 & $21^{\mathrm{o}}$ & Lituânia & 11 \\
\hline 9o & Austrália & 20 & $22^{\circ}$ & Suiça & 10 \\
\hline $10^{\circ}$ - & Brasil & 18 & $23^{\circ}$ & Japão & 9 \\
\hline $11^{\mathrm{o}}$ & Holanda & 16 & $24^{\circ}$ & Coréia do Sul & 9 \\
\hline $12^{\circ}$ & Irã & 15 & 25 o & Irlanda & 8 \\
\hline $13^{\circ}$ & Malásia & 15 & & & \\
\hline
\end{tabular}

Fonte: Dados da pesquisa - Web of Science.

Tabela 5. Principais idiomas das publicações.

\begin{tabular}{llcc}
\hline & Idioma & Publicações & \% \\
\hline $1^{\underline{0}}$ & Inglês & 487 & $92,23 \%$ \\
$2^{\mathbf{o}}$ & Espanhol & 13 & $2,46 \%$ \\
$3^{\mathbf{o}}$ & Português & 6 & $1,14 \%$ \\
$4^{\mathbf{o}}$ & Croata & 5 & $0,95 \%$ \\
$5^{\mathbf{o}}$ & Italiano & 4 & $0,76 \%$ \\
\hline
\end{tabular}

Fonte: Dados da pesquisa - Web of Science.

Os resultados da pesquisa mostram as principais instituições de acordo com o número de publicações que abordam as temáticas estudadas. De acordo com a Tabela 6, cabe ressaltar que a instituição que predomina a produção científica relacionada à 'qualidade de vida' e 'desenvolvimento urbano sustentável' é a Chinese Academy of Sciences, evidenciando as elevadas publicações oriundas do país.

$\mathrm{E}$ ao analisar a Tabela 4 e a Tabela 6, percebe-se que apesar do Brasil se posicionar entre os 10 principais países com publicações nesta temática, nenhuma instituição de ensino ou pesquisa brasileira foi relacionada entre as 25 instituições em destaque.

A fim de verificar a abrangência do tema analisado, são expostas as áreas de pesquisa das publicações relacionadas com os termos 'qualidade de vida' e 'desenvolvimento urbano sustentável'. A Tabela 7, a seguir, apresenta as 25 áreas em destaque nos resultados obtidos neste levantamento.

De acordo com os resultados apresentados na Tabela 7, pode-se perceber que a área de pesquisa com o maior número de publicações, 'Ciência Ambiental e Ecologia' (Environmental Sciences Ecology), está associada aos aspectos ambientais, o que corrobora com $o$ as evidências de uma maior aproximação desses estudos com a temática da sustentabilidade. Destacamse, em seguida, as áreas temáticas 'Engenharia' (Engineering), 'Ciências Tecnológicas e Outros Tópicos' (Science Technology Other Topics) e 'Estudos 
Urbanos' (Urban Studies). Evidencia-se, também, a multidisciplinariedade das pesquisas associadas a 'qualidade de vida' e 'desenvolvimento urbano sustentável', na medida em que abrangem diferentes campos de conhecimento e analisam o fenômeno de diversas perspectivas.

\section{mais citados \\ Características dos 10 artigos}

Nesta fase do estudo, foram selecionadas as dez publicações que obtiveram o maior número de citações, no período de 2008 a 2017, de acordo com o relatório de citações fornecido pelo banco de dados da Web of Science. Esses dados foram obtidos a fim de analisar os principais artigos relacionados aos tópicos "desenvolvimento urbano sustentável" (sustainable urban development) $\mathrm{e}$ "qualidade de vida" (quality of life) que estão sendo estudados nos últimos anos. A seguir, a Tabela 8 apresenta os dados dos artigos mais citados.

Tabela 6. Principais instituições de acordo com o número de publicações.

\begin{tabular}{|c|c|c|}
\hline & Instituição & Publicações \\
\hline $11^{0}$ & Chinese Academy of Sciences & 9 \\
\hline $2^{\mathrm{o}}$ & Vilnius Gediminas Technical University & 8 \\
\hline $3^{0}$ & Polytechnic University of Milan & 6 \\
\hline $4 \underline{0}$ & Universita Mediterranea Di Reggio Calabria & 6 \\
\hline 5 은 & Arizona State University & 5 \\
\hline $6^{0}$ & Consiglio Nazionale Delle Ricerche CNR & 5 \\
\hline $7^{0}$ & Istanbul Technical University & 5 \\
\hline $8^{0}$ & Queensland University of Technology QUT & 5 \\
\hline 9o & Delft University of Technology & 4 \\
\hline $10^{\mathrm{o}}$ & Islamic Azad University & 4 \\
\hline $11^{\underline{0}}$ & Polytechnic University of Timisoara & 4 \\
\hline $12^{\mathrm{o}}$ & Seconda Universita Degli Studi Di Napoli & 4 \\
\hline $13^{0}$ & Tongji University & 4 \\
\hline $14^{\mathrm{o}}$ & University College Dublin & 4 \\
\hline $15^{\mathrm{o}}$ & University of Hong Kong & 4 \\
\hline $16^{\mathrm{o}}$ & University of London & 4 \\
\hline $17^{\mathrm{o}}$ & Aristotle University of Thessaloniki & 3 \\
\hline $18^{\mathrm{o}}$ & Cairo University & 3 \\
\hline 190 & City University of Hong Kong & 3 \\
\hline $20^{\mathrm{o}}$ & Consejo Nacional de Investigaciones Cientificas y Tecnicas Conicet & 3 \\
\hline $21^{\mathrm{o}}$ & Cracow University of Technology & 3 \\
\hline $22^{\underline{0}}$ & Democritus University of Thrace & 3 \\
\hline $23^{0}$ & Huazhong University of Science Technology & 3 \\
\hline $24^{0}$ & Kaunas University of Technology & 3 \\
\hline 25 은 & Liverpool John Moores University & 3 \\
\hline
\end{tabular}

Fonte: Dados da pesquisa - Web of Science. 
Tabela 7. Principais áreas de pesquisa.

\begin{tabular}{|c|c|c|}
\hline & Áreas de pesquisa & Publicações \\
\hline 19 & Ciência Ambiental e Ecologia (Environmental Sciences Ecology) & 170 \\
\hline $2^{\circ} \mathrm{a}$ & Engenharia (Engineering) & 87 \\
\hline 3o & Ciências Tecnológicas e Outros Tópicos (Science Technology Other Topics) & 82 \\
\hline $4 \underline{0}$ & Estudos Urbanos (Urban Studies) & 78 \\
\hline 5 인 & Economia de Negócios (Business Economics) & 49 \\
\hline $6^{0}$ & Arquitetura (Architecture) & 47 \\
\hline $7 \underline{0}$ & Ciências Sociais e Outros Tópicos (Social Sciences Other Topics) & 46 \\
\hline $8^{0}$ & Administração Pública (Public Administration) & 39 \\
\hline 9o & Tecnologia de Construção Civil (Construction Building Technology) & 31 \\
\hline $10^{\mathrm{o}}$ & Geografia (Geography) & 28 \\
\hline $11^{\mathrm{o}}$ & Transporte (Transportation) & 28 \\
\hline $12^{\mathrm{o}}$ & Ciência da Computação (Computer Science) & 27 \\
\hline $13^{0}$ & Combustíveis Energéticos (Energy Fuels) & 25 \\
\hline $14 \underline{0}$ & Recursos Hídricos (Water Resources) & 16 \\
\hline $15^{\mathrm{o}}$ & Educação, Pesquisa Educacional (Education Educational Research) & 15 \\
\hline $16^{\mathrm{o}}$ & Geologia (Geology) & 13 \\
\hline $17^{\circ}$ & Geografia Física (Physical Geography) & 10 \\
\hline $18^{\mathrm{o}}$ & Conservação da Biodiversidade (Biodiversity Conservation) & 9 \\
\hline 19 은 & Ciência de Materiais (Materials Science) & 9 \\
\hline $20^{\circ}$ & Sensoriamento Remoto (Remote Sensing) & 9 \\
\hline $21^{\circ}$ & Sociologia (Sociology) & 9 \\
\hline $22^{\circ}$ & Agricultura (Agriculture) & 8 \\
\hline $23^{0}$ & Artes, Humanidades e Outros Tópicos (Arts Humanities Other Topics) & 8 \\
\hline $24^{\circ}$ & Saúde Ocupacional Pública (Public Environmental Occupational Health) & 8 \\
\hline $25^{\circ}$ & $\begin{array}{l}\text { Ciência da Gestão e Pesquisa Operacional (Operations Research Management } \\
\text { Science) }\end{array}$ & 7 \\
\hline
\end{tabular}

Fonte: Dados da pesquisa - Web of Science.

Tabela 8. Artigos mais citados.

\begin{tabular}{lc}
\hline Publicações & $\begin{array}{c}\text { Número de } \\
\text { Citações }\end{array}$ \\
\hline
\end{tabular}

\section{Artigo 1}

Título: The application of urban sustainability indicators - A comparison between various practices

Autores: Li-Yin Shen; J. Jorge Ochoa; Mona N. Shah; Xiaoling Zhang

Palavras-chave: Urbanização Sustentável; Sustentabilidade Urbana; Indicador; Plano de Governo; Análise Comparativa.

Objetivos: Este trabalho examina nove práticas diferentes que se referem as generalidades e descrições das práticas individuais e à lista completa de indicadores - Lista de Indicadores Internacionais de Sustentabilidade Urbana propostos para monitorar o progresso de cada prática. 0 trabalho propõe uma base comparativa para uma melhor compreensão dos direcionadores e objetivos de cada prática e identifica com quais circunstâncias várias práticas selecionaram seus indicadores. 
Tabela 8. Continuação.

\begin{tabular}{lc}
\hline Publicações & $\begin{array}{c}\text { Número de } \\
\text { Citações }\end{array}$ \\
\hline Áreas Temáticas: Planejamento Urbano Sustentável Ciências Sociais & \\
Resultados: Os resultados da pesquisa mostram como a base comparativa pode & \\
levar ao compartilhamento de conhecimento entre diferentes práticas, o que pode & \\
ser usado para orientar a seleção de indicadores de planos de urbanização & \multirow{2}{*}{144} \\
sustentável e melhorar a comunicação efetiva do status das práticas. O estudo não & \\
apenas revela como diferentes indicadores são selecionados, mas também sugere a & \\
necessidade de processos consistentes de escolha de indicadores com base nos & \\
benchmarks obtidos com as melhores práticas. & \\
\hline
\end{tabular}

\section{Artigo 2}

Título: Assessing the ecosystem service of air pollutant removal by urban trees in Guangzhou (China)

Autores: C.Y. Jim; Wendy Y. Chen

Palavras-chave: Poluição do Ar; Serviço Ecossistêmico; Espaço Verde; Valor Monetário; Mercadoria não Mercantil; Desenvolvimento Sustentável; Ecologia; Urbanforest; Árvore Urbana; Guangzhou; China.

Objetivos: A cidade chave da região do sul da China foi classificada como uma das cidades mais verdes da China e está passando por um rápido crescimento e alguns espaços verdes estão ameaçados pela pressão do desenvolvimento. Além de soluções técnicas para diminuir a poluição do ar, a vegetação urbana é cada vez mais reconhecida como um método alternativo de melhoria, removendo alguns poluentes, principalmente, pelo processo de deposição seca. Este artigo avalia a capacidade e o valor monetário deste serviço ecossistêmico na cidade de Guangzhou, no sul da China.

Áreas Temáticas: Engenharia Ambiental

Resultados: Os resultados indicaram uma remoção anual de $\mathrm{SO}_{2}, \mathrm{NO}_{2}$ e total de partículas suspensas em cerca de $312,03 \mathrm{Mg}$, e os benefícios foram avaliados em RMB 90,19 mil (US \$1,00 - RMB 8,26). Mais remoção foi realizada pelo uso recreativo da terra devido a uma maior cobertura de árvores. Mais concentração de poluentes nos meses secos do inverno induziu maior remoção. 0 custo mais baixo da redução da poluição na China gerou um valor monetário subvalorizado deste benefício ambiental em comparação com os países desenvolvidos.

\footnotetext{
Artigo 3

Título: Ecosystem services and valuation of urban forests in China

Autores: C.Y. Jim; Wendy Y. Chen

Palavras-chave: Floresta Urbana; Serviço de Ecossistemas; Valor Econômico; Valorização Contínua; Preço Hedônico; Valor de Criação; Valor de Humanidade; China.

Objetivos: 0 artigo analisa estudos sobre os múltiplos serviços ecossistêmicos e valores econômicos das florestas urbanas na China, incluindo a melhoria microclimática, o sequestro de dióxido de carbono, a geração de oxigênio, a remoção de poluentes gasosos e particulados, recreação e lazer. Várias técnicas de avaliação foram aplicadas, a maioria das quais ainda está no estágio embrionário.

Áreas Temáticas: Engenharia Ambiental.

Resultados: 0 estudo concluiu que apesar do acúmulo de evidências e descobertas científicas, políticas e práticas de gestão relevantes ainda estão atrasadas, principalmente, por trás do conhecimento. Tanto pesquisadores quanto os praticantes poderiam fazer esforços para manifestar o valor das florestas urbanas e enfatizar as interações mutuamente benéficas entre sociedade humana e natureza. Ainda assim, a compreensão completa de seus serviços ecossistêmicos e valores econômicos apresenta um passo inicial na direção certa.
} 
Tabela 8. Continuação.

\begin{tabular}{lc}
\hline Publicações & $\begin{array}{c}\text { Número de } \\
\text { Citações }\end{array}$ \\
\hline Artigo 4 & \\
Título: Life satisfaction and air quality in London & \\
Autores: George MacKerron; Susana Mourato & \\
Palavras-chave: Satisfação de Vida; Bem-estar Subjetivo; Qualidade & \\
Ambiental; Poluição do Ar; Sistemas de Informação Geográfica; Londres. & \\
Objetivos: Um estudo exploratório que busca avaliar como o uso de dados de & \\
qualidade ambiental em resolução espacial muito alta poderia avançar a literatura \\
empírica examinando as conexões entre os níveis de satisfação com a vida e & \multirow{2}{*}{100} \\
qualidade ambiental, com foco na qualidade do ar. & \\
Áreas Temáticas: Geografia. & \\
Resultados: Os níveis de poluição do ar percebidos e medidos são \\
significativamente negativamente associados à satisfação com a vida dos \\
respondentes da pesquisa, mesmo quando controlam uma ampla gama de outros \\
efeitos. Essas descobertas ainda não podem ser generalizadas com confiança. No \\
entanto, se fossem confirmados por estudos futuros maiores, eles pareceriam \\
fortalecer e ampliar os argumentos existentes em favor de políticas para reduzir a \\
poluição atmosférica urbana.
\end{tabular}

\section{Artigo 5}

Título: From Resilience to Transformation: The Adaptive Cycle in Two Mexican Urban Centers

Autores: Mark Pelling; David Manuel-Navarrete

Palavras-chave: Ciclo Adaptativo; Alterações Climáticas; Gestão de Desastres; México; Poder; Resiliência; Transformação.

Objetivos: Os autores procuraram desenvolver a teoria da resiliência e, em particular, basearam-se na heurística do ciclo adaptativo para ajudar a enquadrar uma análise da transformação social, suas barreiras e motores. Estenderam o trabalho de Scheffer et al. (2002) sobre transformações de domínio, que é útil na introdução da análise de poder interpretada por meio da teoria da estruturação de Giddens (1984). 0 quadro resultante é então aplicado a uma análise comparativa de dois centros urbanos que estão enfrentando impactos diretos e indiretos da mudança climática no caribe mexicano.

Áreas Temáticas: Sociologia.

Resultados: Discussões teóricas mais profundas provocaram questões sobre as dimensões sociais e políticas da resiliência. A lente de resiliência é bem-sucedida em destacar as contradições internas que mantêm as relações sociais de rigidez acima da flexibilidade nos regimes de governança existentes e no caminho do desenvolvimento. Isso gera um conjunto de instituições e ações de reforço que apoiam o status quo, ao mesmo tempo em que minam a flexibilidade de longo prazo, o desenvolvimento equitativo e sustentável. Um dos resultados é a colocação de limites no escopo para adaptação e mitigação às mudanças climáticas, que são externalizações da vida cotidiana e do planejamento do desenvolvimento.

\section{Artigo 6 \\ Título: Sustainability versus liveability: an investigation of neighbourhood satisfaction}

Autores: Peter Howley; Mark Scott; Declan Redmond

Palavras-chave: Política de Cidade Compacta; Desenvolvimento Sustentável; Bairro; Qualidade de Vida.

Objetivos: Combinando dados de pesquisa quantitativa e qualitativa, este artigo avalia a criação de áreas residenciais de alta densidade dentro do centro da cidade e a satisfação dos moradores.

Áreas Temáticas: Administração; Ciências Sociais. 
Tabela 8. Continuação.

\begin{tabular}{lc}
\hline Publicações & $\begin{array}{c}\text { Número de } \\
\text { Citações }\end{array}$ \\
\hline Resultados: Os resultados sugerem que, em muitos casos, não é a alta densidade & \\
que define a fonte de insatisfação dos entrevistados, mas sim, outros fatores & \\
relacionados, como: qualidade ambiental, ruído, falta de envolvimento da & \\
comunidade, tráfego e falta de serviços e instalações. & \\
\hline
\end{tabular}

\title{
Artigo 7
}

Título: Toward Socially Sustainable Urban Transportation: Progress and Potentials

Autores: E. Eric Boschmann; Mei-Po Kwan

Palavras-chave: Acessibilidade Individual; Qualidade de Vida; Equidade Social; Exclusão Social; Transporte Urbano Socialmente Sustentável.

Objetivos: Baseando-se na literatura relevante, este artigo revisa a pesquisa sobre o transporte urbano socialmente sustentável. 0 objetivo deste artigo é explorar a situação atual da pesquisa sobre sustentabilidade social que é particularmente relevante para o transporte urbano. 0 artigo centra-se nas dimensões do transporte urbano socialmente sustentável dentro do contexto das cidades dos EUA.

Áreas Temáticas: Ciências Sociais.

Resultados: 0 transporte urbano socialmente sustentável está implícito na literatura através dos elementos de equidade social, exclusão social e qualidade de vida. Várias áreas de pesquisa bem estabelecidas fornecem um potencial teórico, metodológico e aplicado para o desenvolvimento de futuras agendas de pesquisa de transporte urbano socialmente sustentável, incluindo: forma urbana, acessibilidade baseada em localização e indivíduo e planejamento regional para sustentabilidade.

\author{
Artigo 8 \\ Título: Social Capital and the Quality of Economic Development \\ Autor: Fabio Sabatini \\ Palavras-chave: Não possui.
}

Objetivos: Partindo do pressuposto de que tanto o capital social quanto o desenvolvimento econômico se baseiam em conceitos multidimensionais, esse documento não envolve uma investigação empírica sobre a relação entre os três tipos de capital social até agora identificados pela literatura (ligação, conexão e ligação) e a qualidade do desenvolvimento econômico. A análise baseia-se em um conjunto de dados coletados pelo autor, incluindo cerca de duas centenas de variáveis que representam as diferenças de desenvolvimento econômico e quatro dimensões estruturais do capital social: fortes famílias (geralmente chamadas de capital social vinculado), laços informais fracos (ligação do capital social), organizações voluntárias (vinculando capital social) e participação política ativa.

Áreas Temáticas: Ciências Sociais.

Resultados: Este documento forneceu um mapa das dotações italianas de diferentes tipos de capital social e testou sua relação com a qualidade do desenvolvimento econômico, por meio de análises descritivas e modelos de equações estruturais. Os resultados sugerem que o capital social vinculado tem um efeito negativo significativo no desenvolvimento humano, embora as organizações voluntárias em formação sejam o único tipo de capital social que alimenta o desenvolvimento humano, promovendo assim o crescimento sustentável.

\section{Artigo 9}

Título: Urban growth of Kampala, Uganda: Pattern analysis and scenario development

Autores: Vermeiren Karolien; Van Rompaey Anton; Loopmans Maarten; Serwajja Eria; Mukwaya Paul

Palavras-chave: Expansão Urbana; Regressão Logística; Inexploração das Zonas

Húmidas; Favelas; Planejamento urbano.

Objetivos: 0 artigo tem como objetivo desenvolver um modelo de regressão logística para detectar o crescimento urbano e desenvolver um modelo de expansão urbana. 
Tabela 8. Continuação.

\begin{tabular}{lc}
\hline Publicações & $\begin{array}{c}\text { Número de } \\
\text { Citações }\end{array}$ \\
\hline Áreas Temáticas: Ciências Sociais. & \\
Resultados: Uma validação dos cenários previstos mostrou que o modelo \\
desenvolvido permite prever padrões de expansão urbana com um nível de \\
precisão. Os valores encontrados neste estudo são comparáveis ou ainda mais altos \\
que os valores da aplicação de modelo semelhante em sites europeus de estudo \\
(Poelmans e Van Rompaey, 2009), sugerindo que o crescimento urbano \\
descontrolado é mais fácil de prever do que o controle de crescimento urbano em \\
que planos de zoneamento que, muitas vezes, anulam os principais fatores \\
controladores da expansão urbana. \\
\hline Artigo 10 \\
Título: Residential location and transit-oriented development in a new rail corridor \\
Autores: Doina Olaru; Brett Smith; John H.E. Taplin \\
Palavras-chave: Desenvolvimento Orientado ao trânsito; Decisão de Relocação; \\
Modelos de Escolha Discreta; Variáveis latentes; Aulas latentes. \\
Objetivos: Esta pesquisa avalia como as famílias consideram as características do \\
desenvolvimento orientado para o trânsito em suas decisões de localização com \\
relação às novas estações ferroviárias de Mandu-rah, inauguradas em dezembro de \\
2007 em Perth, Austrália Ocidental. \\
Áreas Temáticas: Ciências Sociais \\
Resultados: Os resultados indicam que a escolha da residência reflete os atributos \\
da vizinhança e da moradia, com significativa heterogeneidade nas populações dos \\
três distritos, em termos de valorização de várias características habitacionais, \\
proximidade de instalações urbanas e transporte. Existe também uma variação \\
significativa nas atitudes das famílias em relação aos ambientes naturais e \\
artificiais. Melhor compreensão das relações complexas entre ambiente, viagens, \\
características sociodemográficas e atitudes domésticas pode ajudar os \\
planejadores a alavancar os benefícios do desenvolvimento orientado para o \\
trânsito e melhorara qualidade do projeto urbano e da vida da comunidade.
\end{tabular}

Fonte: Dados da pesquisa - Web of Science.

No que se refere aos objetivos das dez publicações mais citadas entre os anos de 2008 a 2017, conforme disponibilizado no relatório de citações do banco de dados da Web of Science. A maior parte delas possui abordagem prática (sete artigos), enquanto três delas contam com abordagem teórica.

Além disso, observa-se uma diversidade temática entre os estudos. 0 que corrobora com o referencial teórico apresentado. Segundo Jacobi (2000), a relação entre meio ambiente urbano e qualidade de vida é pensada levando-se em conta aspectos estreitamente relacionados a uma abordagem intersetorial da questão.
Quanto aos estudos práticos, o artigo 6 combina dados de pesquisa quantitativa e qualitativa para avaliar a criação de áreas residenciais de alta densidade dentro do centro da cidade e a satisfação dos moradores. Importante enfatizar que essa combinação de dados quantitativos e qualitativos para análise da qualidade de vida no meio urbano é importante em estudos que procuram explorar a satisfação do indivíduo. Para Zapata e Romero (2010), embora a qualidade de vida possa ser determinada por fatores objetivos, sua manifestação essencial é apreciada na ordem subjetiva, resposta de uma relação dialética entre o objetivo e o subjetivo. 
0 artigo 8 foi desenvolvido a partir de um conjunto de dados coletados pelo autor, esses dados incluem variáveis que representam as diferenças de desenvolvimento econômico e dimensões estruturais do capital social. Tovar (2010) explica que a qualidade de vida é plural e não singular, no sentido de que a qualificação da sensação de existência é criada pelo indivíduo com suas múltiplas determinações geradas pela sua história ontogenia. Levando em consideração além do cenário econômico, os cenários pessoais, familiares, institucionais e comunitários.

Já o artigo 7 centra-se nas dimensões do transporte urbano socialmente sustentável dentro do contexto das cidades, dos Estados Unidos. 0 artigo 10, avalia como as famílias consideram as características do desenvolvimento orientado para o trânsito em suas decisões de localização com relação às novas estações ferroviárias de Mandu-rah, na Austrália Ocidental.

No artigo 4, um estudo exploratório na cidade de Londres examina as conexões entre os níveis de satisfação com a vida e qualidade ambiental, com foco na qualidade do ar. No artigo 5, os autores procuraram desenvolver a teoria da resiliência para ajudar a enquadrar uma análise da transformação social, suas barreiras e motores. Foi aplicado a uma análise comparativa de dois centros urbanos que estão enfrentando impactos diretos e indiretos da mudança climática no caribe mexicano.

0 artigo 2 e 3 foram feitos pelos mesmos autores e ambos tratam de questões climáticas na China. 0 artigo 2, avalia soluções técnicas contra a poluição em Guangzhou, no sul da China. Já o artigo 3, analisa estudos sobre os múltiplos serviços ecossistêmicos e valores econômicos das florestas urbanas na China.

Os artigos citados acima analisam as temáticas de desenvolvimento urbano sustentável e qualidade de vida nas suas mais diferentes práticas, eles passam por assuntos, como: transporte sustentável, trânsito, qualidade do ar, floresta urbana, mudanças climáticas e poluição, dos mais variados países. 0 que vai ao encontro com o que Turkoglu (2015) expõe: aqueles interessados nos estudos sobre cidades e meio ambiente em geral devem estar conscientes de que vivemos em diferentes lugares, cada um dos quais tem numerosos atributos ambientais e esses lugares também são importantes para a nossa qualidade de vida.

Quanto aos artigos 1 e 9, o artigo 1 propõe uma base comparativa que pode levar ao compartilhamento de conhecimento entre diferentes práticas, $o$ que pode ser usado para orientar a seleção de indicadores de planos de urbanização sustentável e melhorar a comunicação efetiva do status das práticas. Por fim, o artigo 9 teve como objetivo desenvolver um modelo de regressão logística para detectar 0 crescimento urbano e desenvolver um modelo de expansão urbana.

Como Kamp et al. (2003) observa: problemas típicos da grande cidade, como a segregação, a degradação da vizinhança e o aumento do tráfego rodoviário são questões políticas centrais e assuntos discutidos entre os dez artigos mais citados. 0 que permite entender a importância do desenvolvimento urbano sustentável e da qualidade de vida em muitas áreas. E ainda, a importância desses dois temas unidos para a evolução de cidades sustentáveis nos mais diversos lugares do planeta.

\section{Considerações finais}

0 presente estudo teve por objetivo identificar as principais características da produção científica internacional relacionada aos termos "desenvolvimento urbano sustentável" e "qualidade de vida", nos últimos dez anos. Assim, foi desenvolvido um estudo bibliométrico, por meio da base de dados Web of Science, relacionando os tópicos citados, identificando 528 publicações, 
das quais destacam-se os artigos científicos que correspondem a 51\% das publicações, seguido dos papers publicados em anais, com $46 \%$ das publicações.

Entre os principais resultados, pode-se destacar que as temáticas analisadas são emergentes e crescem sua produção a cada ano. Em relação as principais fontes, destacam-se os seguintes Journals: 'Procedia Engineering', 'Procedia Social and Behavioral Sciences' e 'Sustainability'. Quanto às instituições, Chinese Academy of Sciences apresenta maior representatividade, evidenciando as elevadas publicações originadas do país.

Em sua maioria, os estudos são provenientes da China e da Itália e estão associados às áreas de pesquisa 'Ciência Ambiental e Ecologia' (Environmental Sciences Ecology), 'Engenharia' (Engineering). Observa-se a predominância dos estudos oriundos da China, Itália e Estados Unidos e, ainda, a presença do Brasil neste ranking, ocupando a $10^{\mathrm{a}}$ posição entre os países com maior número de publicações nesta área. Nesse sentido, evidencia-se a preocupação com aspectos ambientais e de planejamento relacionados à temática da sustentabilidade atrelada ao desenvolvimento urbano e a qualidade de vida.

Ao analisar os dez artigos mais citados, demonstrou-se a multidisciplinaridade das temáticas, surgindo em diversas e distintas áreas, nos mais variados assuntos. 0 que comprova a amplitude do tema. Ainda, as cidades e países predominantes no foco desses estudos estão localizados, principalmente, no continente europeu, além de Estados Unidos, sendo estes países com maior desenvolvimento urbano. Também, destaca-se a dimensão ambiental sobreposta às dimensões social e econômica na análise dos mais citados, tendo como possível explicação que esses países apresentam maiores problemas ambientais em relação aos problemas sociais ou econômicos para serem debatidos.

Como limitações do estudo, considera-se $o$ fato deste ter sido pesquisado em apenas uma base de dados. Sugere-se, portanto, a realização de outras incursões de pesquisa maior amplitude.

Ressalta-se, por fim, que os resultados desta pesquisa são relevantes, portanto, para a construção do conhecimento científico sobre os assuntos tratados. A pesquisa bibliométrica permitiu ampliar a compreensão dos temas e abordagens utilizadas na construção deste campo de conhecimento. Possibilitou, ainda, a identificação dos estudos associados à sustentabilidade na temática abordada, com vistas ao ambiente urbano e a qualidade de vida e como essa combinação impacta no desenvolvimento sustentável.

\section{Conflito de interesses}

Os autores declaram não haver conflito de interesses.

\section{Referências}

Bartley, M.; O’Neill, D. Transportation and driving in longitudinal studies on ageing. Age and Ageing, v. 39, p.631-636, 2010. https://doi.org/10.1093/ageing/afq089

Cortese, T. T. P.; Kniess, C. T.; Maccari, E. A. Cidades inteligentes sustentáveis. São Paulo: Malone, 2017.

Duran, J.; Pérez, V. Smart, innovative and sustainable cities for the future income: Caracas city. In Proceeding of the XXXV Central American and Panama Convention, IEEE, p. 1-6, 2015.

Gehl, J. Cidades para Pessoas. 2. ed. São Paulo: Perspectiva, 2013.

Giddings, B.; Hopwood, B.; Mellor, M.; O'Brien, G. Back to the city: a route to urban sustainability. In: Jenks, M; Dempsey, N. Future forms and design for sustainable cities. Amsterdan: Architectural, 2005.

Han, J.; Fontanos, P.; Fukushi, K.; Herath, S.; Heeren, N.; Naso, V.; Cecchin, C.; Edwards, P.; Takeuchi, K. Innovation for sustainability: 
toward a sustainable urban future in industrialized cities. Sustainability Science, v. 7, Suppl. 1, p.91-100, 2012. https://doi.org/10.1007/s11625-011-01522

IPCC - Intergovernmental Panel on Climate Change. Climate change 2007: synthesis report. 2007. Disponível em: <http://www.ipcc.ch/publications_and_data/ ar4/syr/en/contents.html>. Acesso em: 04 jul. 2018.

Jacobi, P. Cidade e meio ambiente: percepções e práticas em São Paulo. São Paulo: Annablume, 2000.

Kamp, I.; Leidelmeijer, K.; Marsman, G.; Hollander, A. Urban environmental quality and human well-being Towards a conceptual framework and demarcation of concepts; a literature study. Landscape and Urban Planning, v. 65, n. 1/2, p. 5-18, 2003. https://doi.org/10.1016/S0169-2046(02) 00232-3

Kilinc, M. Institutional environment and place attachment as determinants of elders' life satisfaction. Ankara: Middle East Technical University, 2006. (Dissertation).

Kobayashi, K. R. A.; Kniess, T. C.; Serra, R. A. F.; Ferraz, N. R., R.; Ruiz, S. M. Cidades Inteligentes e Sustentáveis: estudo bibliométrico e de informações patentárias. International Journal of Innovation, v. 5, n. 1, p. 77-96, 2017. https://doi.org/ 10.5585/iji.v5i1.159

Kran, F.; Ferreira, F. P. M. Qualidade de vida na cidade de Palmas-TO: uma análise através de indicadores habitacionais e ambientais urbanos. Ambiente \& Sociedade, v. 9, n. 2, p. 123-141, 2006. https://doi.org/10.1590/ S1414-753X2006000200007

Leite, C.; Awad, J. C. M. Cidades sustentáveis, cidades inteligentes: desenvolvimento sustentável num planeta urbano. Porto Alegre: Bookman, 2012.

López-Vizcaíno, M. E.; Sánchez-Fernádez, P. La medición de la calidad de vida en las comarcas gallegas. Revista Galega de Economía, v. 18, n. 1, p.1-20. 2009. Disponível em: <http://www.usc.es/econo/ RGE/Vol18_1/castelan/art2c.pdf>. Acesso em: 04 jul. 2018.

Macedo, M. A. S.; Casa Nova, S. P.; Almeida, K. Mapeamento e análise bibliométrica da utilização da análise envoltória de dados (DEA) em estudos das áreas de contabilidade e administração. Contabilidade, Gestão e
Governança, v. 12, n. 3, p.87-101, 2009. Disponível em: <https://cggamg.unb.br/index.php/contabil/article/view /92/pdf_119>. Acesso em: 04 jul. 2018.

Oliveira, B. G.; Souza-Lima, E. J. 0 desenvolvimento sustentável em foco: uma contribuição multidisciplinar. 1 ed. São Paulo: Annablume, 2006.

Programa Cidades Sustentáveis. Programa Cidades Sustentáveis. São Paulo: Rede Nossa São Paulo; Rede Social Brasileira por Cidades Justas e Sustentáveis; Instituto Ethos. $2018 . \quad$ Disponível em: <http://www.cidadessustentaveis.org.br>. Acesso em: 20 mar. 2018.

Rogers, R. Prólogo. In: Gehl, J. Cidades para pessoas. 2 ed. São Paulo: Perspectiva, 2013.

Rostaing, H. La bibliométrie et sés techniques. Toulouse: Sciences de la Société. Marseille: Centre de Recherche Rétrospective de Marseille, 1997.

Silva, M. R. Análise bibliométrica da produção científica docente do programa de pós-graduação em educação especial/ UFSCar: 1998-2003. São Carlos: Universidade Federal de São Carlos, 2004. (Dissertação).

Tovar, A. C. T. Calidad de vida: realidad y percepción. Bogotá: Universidad Nacional de Colombia, 2010.

Turkoglu, H. Sustainable development and quality of urban life. Procedia - Social and Behavioral Sciences, v. 202, p. 10-14, 2015. https://doi.org/10.1016/j.sbspro.2015.08.20 3

Ülengin, B.; Ülengin, F.; Güvenç, U. A multidimensional approach to urban quality of life: The case of Istanbul. European Journal of Operational Research, v. 130, n. 2, p. 361-374, 2001. https://doi.org/ 10.1016/S0377-2217(00)00047-3

Wahl, H. W.; Weisman, G. D. Environmental gerontology at the beginning of the new millenium: reflections on its historical empirical and theoretical development. The Gerontologist, v. 43, n. 5, p. 616-627, 2003. https://doi.org/10.1093/geront/43.5.616

Węziak-Białowolska, D. Quality of life in cities: Empirical evidence in comparative European perspective. Cities, v. 58, p. 87-96, 2016. https://doi.org/10.1016/j.cities.2016. 05.016

Williams, K. Sustainable cities: research and practice challenges. International Journal of 
Urban Sustainable Development, v. 1, n. $1 / 2$, p. 128-132, 2010. https://doi.org/ $10.1080 / 19463131003654863$

Zapata, C. S.; Romero, H. G. Calidad de vida y factores asociados en mujeres con cáncer de mama en Antioquia, Colombia. Revista Panamericana de Salud Pública, v. 8, n. 1, p. 9-18, 2010. Disponível em: <https://www.scielosp.org/article/rpsp/201 0.v28n1/9-18/>. Acesso em: 20 mar. 2018. 